\title{
Prospects to study the Ice Giants with the ngVLA
}

A white paper for the 2023-2031 Decadal Survey on Planetary Science and Astrobiology

\author{
Principal Author: \\ Name: Imke de Pater \\ Institution: University of California, Berkeley \\ Email: imke@ berkeley.edu \\ Phone: 510-642-5275
}

\section{Co-authors:}

Chris Moeckel - University of California, Berkeley

Joshua Tollefson - University of California, Berkeley

Bryan Butler - National Radio Astronomy Observatory

Katherine de Kleer - California Institute of Technology

Leigh Fletcher - Leicester Univ, UK

Mark A. Gurwell - Center for Astrophysics/Harvard \& Smithsonian

Statia Luszcz-Cook - Columbia Univ.

Stefanie Milam - NASA Goddard Space Flight Center

Edward Molter - University of California, Berkeley

Arielle Moullet - SOFIA

R. J. Sault - University of Melbourne

Thomas R. Spilker - Independent consultant

\section{Endorsements}

Shahid Aslam - (NASA Goddard Space Flight Center); Gordon Bjoraker - (NASA Goddard Space Flight Center); Richard Cartwright - (SETI Inst.); Martin Cordiner - (NASA GSFC/Catholic University of America); Richard Cosentino - Univ. Maryland/NASA Goddard); David DeBoer - (UC Berkeley); Stephen H. Brecht - (Bay Area Research Corporation, Orinda, CA/USA); Peter Gao - (UC Berkeley); Charles Goullaud - (UC Berkeley); Mark Hofstadter (JPL/CIT); Joseph Lazio - (JPL/CIT); Cheng Li - (UC Berkeley); Eric Murphy - (NRAO);

Quentin Nenon - (SSL/UC Berkeley); Glenn Orton - (JPL/CIT); Marzia Parisi - (JPL/CIT); Carol Paty - (Univ. of Oregon); Erin Redwing - (UC Berkeley); Kirby D. Runyon - (JHU/APL); Abigail Rymer - (JHU/APL); Kunio Sayanagi - (Hampton Univ.); James Sinclair - (JPL/CIT); Krista Soderlund - (Univ. Texas); Linda Spilker - (JPL/CIT); Geronimo Villanueva - (NASA Goddard Space Flight Center); Eric Villard - (ALMA/Chile); Michael H. Wong - (UC Berkeley) 


\section{Summary}

The deep atmospheres of the giant planets provide crucial constraints on their composition, an essential parameter in planet formation models. These deep atmospheres can only be probed remotely at radio wavelengths. The next-generation Very Large Array (ngVLA) will enable the highest sensitivity and spatial resolution observations at wavelengths of $0.25-26 \mathrm{~cm}(1.2-116 \mathrm{GHz})$, enabling one to derive the composition and atmospheric dynamics of the Ice Giants Uranus and Neptune from the stratosphere down to tens of bars, and constrain $\mathrm{H}_{2} \mathrm{O}$ at 100s of bars via mmspectroscopy of CO. A comparison of the ngVLA with a future Ice-Giant mission shows that the ngVLA will provide a spatial resolution at wavelengths $\lesssim 10 \mathrm{~cm}$ that is superior over radio instrumentation on an Ice-Giant mission. The ngVLA will provide unique scientific observations (e.g., full maps over a broad wavelength range, and $\mathrm{mm}$-wavelength spectroscopy), providing invaluable support to space missions. It also is a potential ground station for spacecraft telemetry.

\section{Introduction}

Comparative planetology of the bulk composition is a key ingredient in the development of models to explain the formation and evolution of our Solar System. For the giant planets, measurements of the noble gases and condensable species $\left(\mathrm{CH}_{4}, \mathrm{NH}_{3}, \mathrm{H}_{2} \mathrm{~S}, \mathrm{H}_{2} \mathrm{O}^{1}\right)$ provide the essential compositional information. Only an atmospheric entry probe can measure the noble gases, and such a probe can determine the temperature profile and composition at its location of entry, as done by the Galileo Probe on Jupiter down to $\sim 20$ bar [25]. The depth a probe can sample depends on the opacity of the atmosphere at the data relay link frequency and the amount of time the orbiter is within the probe's antenna beam to receive the signal. The latter limits the depth a probe can reach to no more than $\sim 10$ bar on the Ice Giants. A direct link from the probe to Earth requires not only that the probe be visible from Earth during descent, but extremely large antennas are needed to receive the probe's (typically at $\sim 100$ bits per second) signal; antennas with effective sizes (100's $\mathrm{km}^{2}$ ) that are well beyond those currently envisioned. Hence a probe will only reach depths on the Ice Giants below its top-most $\left(\mathrm{CH}_{4}\right)$ cloud layer, leaving composition essentially unsolved.

In this White Paper we discuss how the next-generation Very Large Array (ngVLA) can help address the two fundamental questions: 1. How did our Solar System form and evolve? 2. How does a planet's general circulation affect composition at and above the cloud layers?

The ngVLA is a synthesis radio telescope sensitive to wavelengths $\lambda=0.25-26 \mathrm{~cm}(1.2-116$ $\mathrm{GHz}$ ), which can operate in a phased or interferometric mode. The array will have $\sim 214$ reflector antennas, each $18 \mathrm{~m}$ in diameter, as well as a dense core of $196-\mathrm{m}$ antennas that will be sensitive to larger angular scales that are not detected by the main array. The ngVLA will be $\sim 10$ times more sensitive and provide $\gtrsim 10$ times higher spatial resolution than the VLA and Atacama Large (sub)Millimeter Array (ALMA) [21].

We focus our discussion on the Ice Giants, bodies that are common amongst exoplanets [e.g., 18], and which to date are far from understood. Deriving composition on any giant planet requires

\footnotetext{
${ }^{1}$ The cloud layers are: a deep water (solution) cloud topped off with $\mathrm{H}_{2} \mathrm{O}$ ice; an $\mathrm{NH}_{4} \mathrm{SH}$ cloud above which only $\mathrm{NH}_{3}$ or $\mathrm{H}_{2} \mathrm{~S}$ gas remains, which condenses into its own ice-cloud at higher altitudes; on Uranus and Neptune the top-most cloud layer is $\mathrm{CH}_{4}$-ice. See Fig. $3 \mathrm{~b}$ for a schematic.
} 

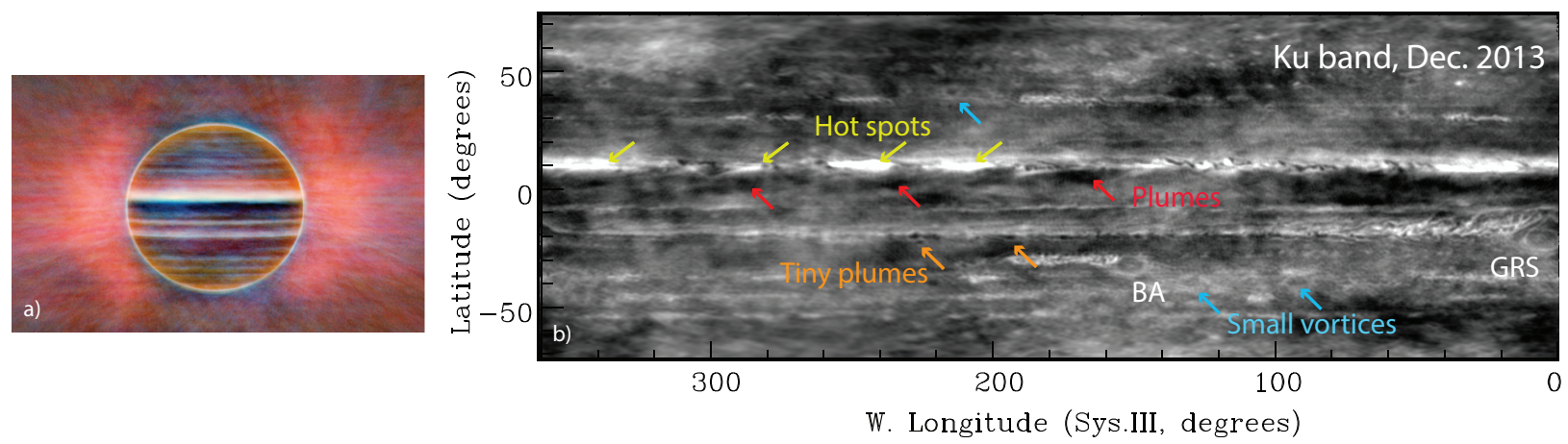

Figure 1: a) VLA radio map of Jupiter constructed from several 10-hr observing sessions at three wavelengths, after subtraction of a uniform limb-darkened disk: $2 \mathrm{~cm}$ (blue), $3.5 \mathrm{~cm}$ (gold), and $6 \mathrm{~cm}$ (red). Any longitudinal structure is smeared by the planet's rotation. [5]. b) Longitude-resolved map from panel a) at 2 $\mathrm{cm}(\mathrm{Ku}$ band, 12-18 GHz). Bright features indicate a high brightness temperature. Much finescale structure can be discerned. The GRS and Oval BA are indicated, as well as hot spots (yellow arrows), ammonia plumes (red), small vortices (cyan), and tiny ammonia plumes (orange) [7].

measurements of the altitude-dependent profiles of the condensible species at depth across the disk; such profiles also contain clues to the general circulation, essential to interpret composition at depth.

Below we highlight recent VLA results on Jupiter and the Ice Giants, and discuss how the latter will be improved with the ngVLA [6]. We end the paper with a comparison of these results with potential spacecraft missions equipped with instruments like the Microwave Radiometer (MWR) on Juno. We will briefly address use of the ngVLA to communicate with deep space spacecraft.

\section{Ground-based Radio Observations of the Giant Planets}

\subsection{Deep Tropospheres using Continuum Wavelengths}

At the radio wavelengths that probe the deep atmospheres of the giant planets, we observe the thermal (blackbody) radiation emitted by the atmosphere. The sources of opacity at these wavelengths are collision induced absorption (CIA) by $\mathrm{H}_{2}$, and absorption by the trace (condensable) gases $\mathrm{NH}_{3}, \mathrm{H}_{2} \mathrm{~S}, \mathrm{PH}_{3}$, and $\mathrm{H}_{2} \mathrm{O}$ [7]. For near-solar-composition atmospheres, like Jupiter and Saturn, most of the atmospheric radio opacity has been attributed to ammonia gas. Radio spectra of Uranus and Neptune, however, show that there is a deficiency of $\mathrm{NH}_{3}$ gas [10]. The $\mathrm{S} / \mathrm{N}$ ratio on these planets appears to be considerably (factor of $~ 30$ ) enhanced above the solar value, and the main source of opacity on these planets is likely $\mathrm{H}_{2} \mathrm{~S}$ gas $[3,17,23]$.

The thermal emission from all four giant planets has been imaged with the VLA. To construct high signal-to-noise maps, one needs to integrate over several hours, resulting in maps that are smeared in longitude and only reveal brightness variations in latitude. In order to discern longitudinal structures on radio maps, such as the Great Red Spot (GRS) on Jupiter, we developed an algorithm to take out a planet's rotation without significantly degrading its sensitivity [20]. This algorithm has been applied to VLA data of all planets, and have revealed compositional variations that show dynamical systems over a range of spatial scales $[6,7,17]$. 


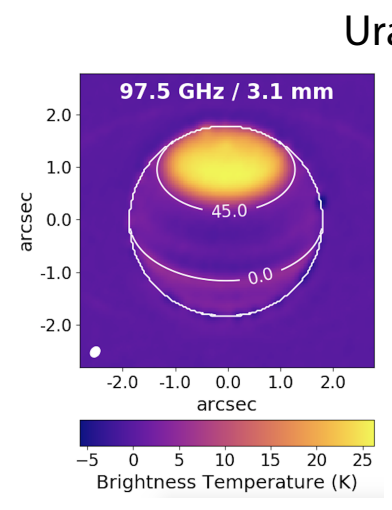

Uranus
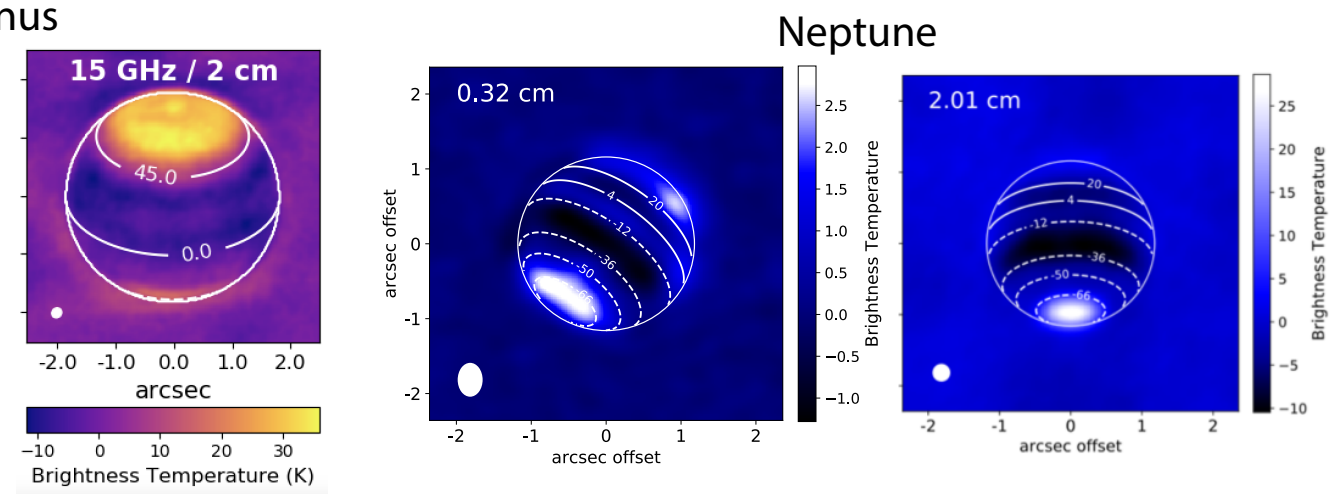

Figure 2: Left: Longitude-smeared maps of Uranus from ALMA (3 mm), and the VLA $(2 \mathrm{~cm})$ [17]. Right: Longitude-smeared maps of Neptune from ALMA $(3 \mathrm{~mm})$ and the VLA $(2 \mathrm{~cm})$. A uniform limb-darkened disk was subtracted to enhance the contrast on the disk. The white ellipse shows the beam. [23]

Jupiter. Fig. 1a shows a longitude-smeared composite multi-wavelength radio map of Jupiter, revealing fine banded structure across the disk. The pink glow surrounding the planet is synchrotron radiation produced by spiraling electrons trapped in Jupiter's magnetic field. The bright bands are roughly co-located with the brown belts seen at visible wavelengths. A longituderesolved map is shown in panel b, at a typical spatial resolution of 1000-1200 km, which is close to Juno's MWR spatial resolution near perijove. The observed variations in brightness are due to spatial variations in $\mathrm{NH}_{3}$ gas, caused by a combination of atmospheric dynamics and condensation at higher altitudes. These "weather" phenomena are mostly confined to pressures $P \lesssim 2-3$ bar [5].

Spectra of zones, belts, plumes and hot spots have been analyzed with radiative transfer (RT) calculations to determine the altitude distribution of $\mathrm{NH}_{3}$ gas at these locations. $\mathrm{NH}_{3}$ is brought up from the deep atmosphere to the cloud condensation levels in the Equatorial Zone (EZ), in particular in the large plumes (red arrows in Fig. 1b), and dry air is descending in the hot spots, and in the North Equatorial Belt (NEB) down to at least 20 bar. The hot spots are also bright at wavelengths of $5 \mu \mathrm{m}$, and are confined to pressures $\lesssim 8-10$ bar [5,7,9]; the plumes have been hypothesized to form the counterpart of the equatorial Rossby wave [5] theorized to produce the 5 $\mu \mathrm{m}$ hot spots [22].

Uranus \& Neptune. Fig. 2 shows longitude-smeared maps of the Ice Giants. No longitudinal structure can be discerned above the noise level in longitude-resolved maps $[6,17]$. Both planets have radio-bright poles (only the $\mathrm{S}$. pole has been seen on Neptune, though), indicative of a very low radio opacity down to $\sim 40-50$ bar (see Fig. 3), so that deep warm layers in the atmosphere are probed. The poles are also enhanced in brightness in the mid-IR, attributed to adiabatic heating through compression in the stratosphere and upper troposphere. The combination of near-IR, midIR and radio data suggests a global circulation pattern where air is rising above southern and northern midlatitudes from the troposphere up well into the stratosphere, and subsidence of dry air over the pole down to 40-50 bar and over the equator $[4,11,17,19,23]$. This model, however, does not explain all observations, which has led to hypothesized systems of stacked circulation cells [8]. 

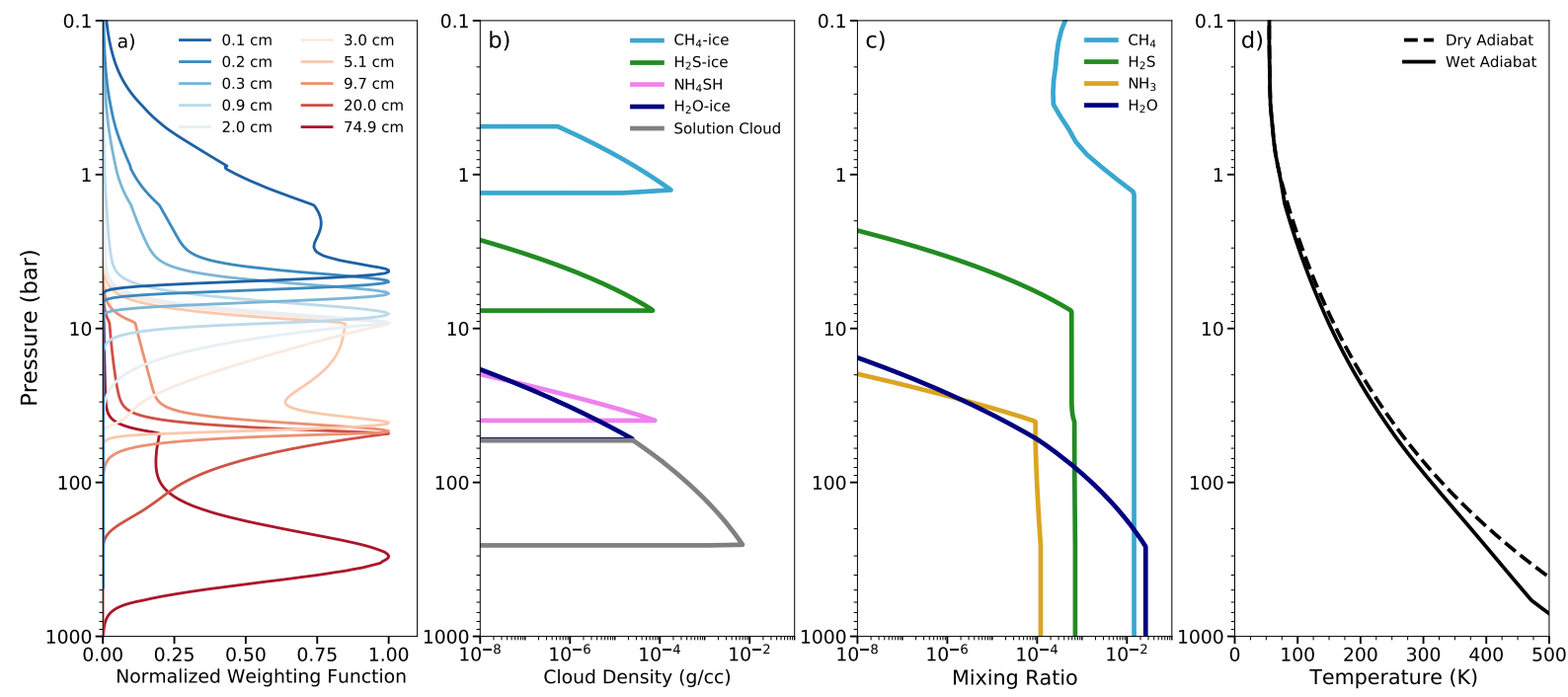

Figure 3: a) Normalized weighting functions for Neptune at nadir for different wavelengths. Weighting functions are calculated based on the gas and temperature profiles; those shown in panel a are based on the gas profiles shown in panel $\mathrm{c}$ and thermal profiles shown in panel d. Since these profiles are compositiondependent, a decrease in $\mathrm{H}_{2} \mathrm{~S}$ abundance would push the weighting functions to deeper pressures. b) Cloud density expected for the gas profiles in panel c (assuming thermo-chemical equilibrium). c) Gas profiles in this study, which are based on $30 \times$ protosolar $\mathrm{CH}_{4}, \mathrm{H}_{2} \mathrm{~S}$, and $\mathrm{H}_{2} \mathrm{O}$, and $1 \times$ protosolar $\mathrm{NH}_{3}$ in the deep atmosphere. These profiles provide a best fit to Neptune's radio spectrum. d) Dry and wet adiabatic temperature profiles used in this study. [23]

\subsection{Stratospheres and Disequilibrium Species using mm-Spectroscopy}

Disequilibrium species provide insight into a planet's chemistry and dynamics at altitudes that cannot be probed otherwise. A prime example is $\mathrm{PH}_{3}$ on Jupiter, a quasi-conserved tracer of dynamical motions, being advected or mixed from the deeper troposphere faster than it can be destroyed chemically. It therefore acts as a measure of the strength of vertical motions, as well as the efficiency of photolytic destruction in the upper troposphere. On the Ice Giants we are particularly interested in CO, which was detected in Neptune's stratosphere in the early 1990s with the single dish radio telescope JCMT at $\sim 0.9 \mathrm{~mm}$, with an abundance $\sim 1000$ times higher than predicted from thermochemical models [16]. Other species, e.g., hydrocarbons $\mathrm{C}_{x} \mathrm{H}_{y}, \mathrm{HCN}$, and $\mathrm{H}_{2} \mathrm{O}$, have also been detected in planets' stratospheres, and may have formed through photochemistry (e.g., $\mathrm{C}_{x} \mathrm{H}_{y}$ from methane gas), or result from infalling materials (e.g., comets, micrometeorites, rings/satellites). $\mathrm{CO}$ in the stratosphere can have a similar external origin, or could be brought up from the deep atmosphere. $\mathrm{CO}, \mathrm{HCN}$, and $\mathrm{H}_{2} \mathrm{O}$ have rotational transitions at $\lambda \gtrsim 2.6 \mathrm{~mm}$, i.e., within the ngVLA wavelength range.

If $\mathrm{CO}$, or a fraction thereof, has an internal origin, it can be used to constrain the planet's deep water abundance, since $\mathrm{CO}$ is produced from $\mathrm{CH}_{4}+\mathrm{H}_{2} \mathrm{O} \rightarrow \mathrm{CO}+3 \mathrm{H}_{2}$. Using this idea together with a variety of eddy diffusion coefficients, the water abundance in Neptune has been shown to possibly be enriched a few 100 times solar $[2,15]$. 


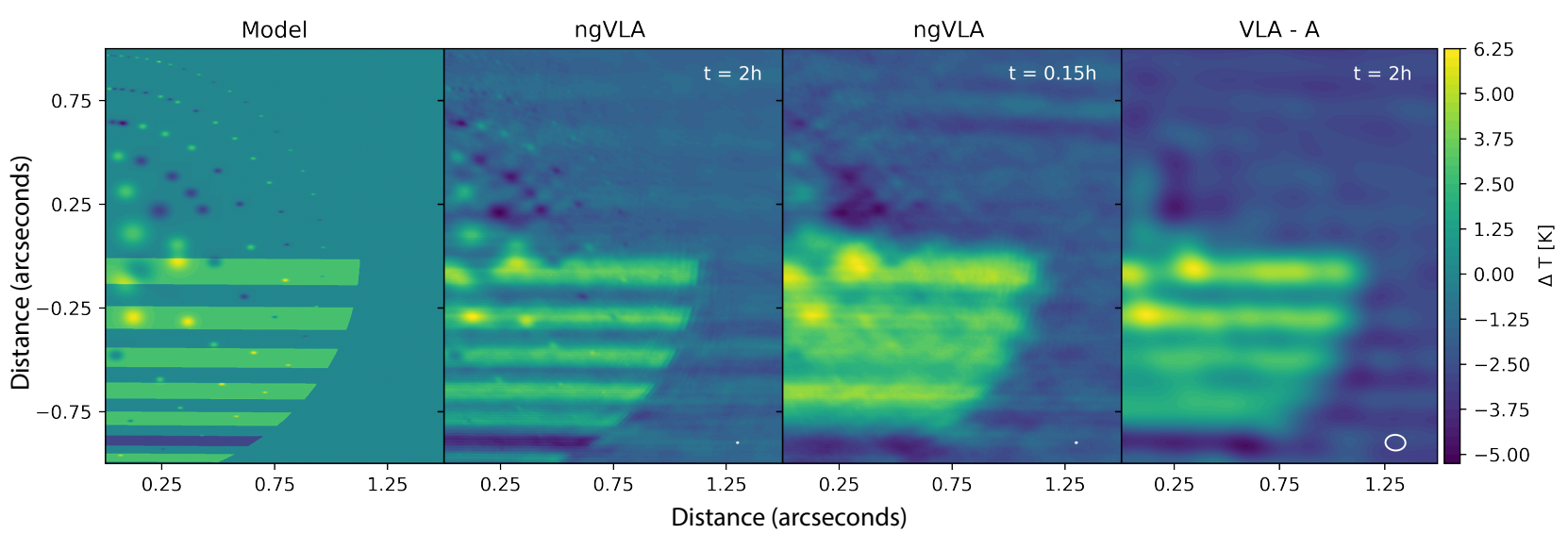

Figure 4: a) A toy model of Neptune after subtraction of a 120-K limb-darkened disk. Note the bright and dark stripes in the southern hemisphere and cold \& hot spots near the center, extending up into the northern hemisphere. The brightness temperature of the various features varies from $\sim 2$ up to $6 \mathrm{~K}$. b) Toy model as seen with the ngVLA after an integration time of $2 \mathrm{hrs}$. c) Toy model as seen with ngVLA after integrating for 10 min. d) Toy model as seen with the VLA in its A configuration, after integrating for 2 hrs. Maps with integration times of $2 \mathrm{hrs}$ do need to be created using using the derotation technique [20]. The beam size is shown as a white oval in the bottom right of each simulation (miniscule, 0.006", for the ngVLA). (simulations by Moeckel \& Tollefson.)

\section{3 ngVLA Potential to Sound the Ice Giants' Atmospheres}

Continuum wavelengths. To highlight the capabilities of the ngVLA, we have constructed a toy (artificial) model of Neptune at $\sim 0.75 \mathrm{~cm}(40 \mathrm{GHz})$ with jovian-like zones and belts, (cold) plumes and (warm) hotspots, with a contrast varying from $\sim 2$ up to $6 \mathrm{~K}$ (compared to a $\sim 120 \mathrm{~K}$ disk temperature) (Fig. 4a). We used the SIMOBSERVE routine in the Common Astronomy Software Applications package, CASA, to simulate what would be seen with the VLA in its most-extended (A) configuration at a spatial resolution of $0.1 "$ ( $~ 2000 \mathrm{~km}$ on Neptune; Fig. 4d), and with the full ngVLA (at 0.006" ( $130 \mathrm{~km})$, Fig. 4b,c). After integrating for $2 \mathrm{hrs,} \mathrm{the} \mathrm{ngVLA} \mathrm{is} \mathrm{able} \mathrm{to} \mathrm{pick}$ up all small sources from the model, i.e., localized storms less than $0.01 "(\sim 200 \mathrm{~km})$ in size, due to its high sensitivity and superb $u v$-coverage. Clearly, with multifrequency maps one can create latitude profiles of condensable species similar to those on Jupiter [5,7,14]. Such profiles will help decipher the dynamics in the Ice Giants' atmospheres, such as the potential vertically stacked circulation cells [8]. In contrast to the present ALMA/VLA maps [17,23], the ngVLA will also be sensitive enough to map vortices, analogous to e.g., the GRS, Oval BA and smaller vortices on Jupiter (Fig. 1). Such data on Uranus and Neptune will reveal, for example, the depth and altitude range of dark spots, such as Neptune's Great Dark Spot imaged with the Voyager 2 spacecraft. Dark spots on Neptune appear every $\sim 5$ years and may last for several years [12]; they are visible at blue wavelengths, and based upon their wind speed might be confined to deeper layers in the atmosphere; however, we need the ngVLA to determine their vertical extent, crucial information to model these still-mysterious structures. Radio observations of vortices and the atmospheric structure below the visible storm systems are essential to model the planets' weather layers and address static stability [24] in the atmosphere. Such information is needed to get a good handle on the planets' deep atmospheric dynamics and composition. 
Millimeter spectroscopy. As shown in Figure 3, even at long wavelengths it will be hard to constrain the water abundance. However, as shown in Section 2.2, an accurate measurement of $\mathrm{CO}$ can constrain the water abundance, given good estimates of the eddy diffusion coefficient (measurements of disequilibrium species will help here). The CO 1-0 line is the most sensitive to a potential internal source since the wings of the line, seen in absorption, originate relatively deep in the troposphere, in contrast to the stratospheric emission line at its center. One of the challenges is to observe the entire line, which blends in with the background emission many, perhaps $\sim 10, \mathrm{GHz}$ from line center [15]. Hence one needs to map a very broad line $(\sim 105-116 \mathrm{GHz})$ at low spectral resolution, simultaneously with the narrow emission peak $(\lesssim 10 \mathrm{MHz})$ at high resolution. This was not possible in previous studies, and ALMA's 2-GHz broad bandwidth is too small to capture the entire line. With the ngVLA we will be able to observe the entire line over at least $\sim 10 \mathrm{GHz}$, while spectrally resolving its narrow peak.

\section{Comparison with Missions}

Jupiter and Juno. While the atmosphere of Jupiter can be probed down to the $\sim 10$ bar level with the VLA, probing deeper levels requires wavelengths $\gtrsim 6 \mathrm{~cm}$, which is next to impossible to do from the ground because the signal is overwhelmed by Jupiter's synchrotron radiation (Fig. 1a). These deeper layers have been probed with MWR on the Juno spacecraft, which orbits Jupiter along polar orbits with a perijove inside the radiation belts. MWR mapped Jupiter's brightness temperature along narrow swaths of longitude along these tracks. While Juno confirmed the large variability in brightness temperature at pressures $\lesssim 3$ bar (the weather layer, mentioned above), it was able to characterize the latitudinal structure down $\gtrsim 100$ bar, and found that $\mathrm{NH}_{3}$ gradients extend to at least a few tens of bars over the entire planet $[1,14]$.

Ice Giants. Let us compare the potential of the ngVLA with a MWR-like instrument on a space mission to the Ice Giants. Typical resolutions with MWR on Juno vary from $~ 1000$ to $2000 \mathrm{~km}$ at closest approach, at a typical altitude of $\sim 4000 \mathrm{~km}$. Around $1 \mathrm{~cm}(30 \mathrm{GHz})$, the spatial resolution of the ngVLA is almost an order of magnitude higher than MWR: $\sim 0.006$ " ( $~ 130 \mathrm{~km}$ on Neptune, $90 \mathrm{~km}$ on Uranus), and varies linearly with inverse wavelength. At $20 \mathrm{~cm}(1.5 \mathrm{GHz})$, the spatial resolution is $\sim 0.2^{\prime \prime}$ (3600 km on Neptune; $2400 \mathrm{~km}$ on Uranus), which translates roughly into $8^{\circ}$ $\left(6^{\circ}\right)$ on Neptune (Uranus) at disk-center. Since neither planet has synchrotron radiation, the deep atmospheres can be probed from the ground without interference of nonthermal emissions. The advantage of a MWR on an Ice-Giant mission is absolute calibration (on Juno the calibration is accurate to within $2 \%$, compared to $\sim 5 \%$ from the ground), access to higher latitudes (if in a polar orbit) and a higher spatial resolution at the longest wavelengths. However, MWR has to be so close to Neptune to achieve this high resolution that one never gets a global map; only the ngVLA can provide such maps. MWR also lacks the spectral information at mm-wavelengths. Clearly, observational support from the ngVLA would considerably enhance the science return from an Ice-Giant mission.

Telemetry. The ngVLA may also provide a very sensitive ground-based station to conduct telemetry, tracking, and to communicate with deep space spacecraft [13]. This is not a new idea: for example, in the 1980's the VLA was used to receive signals from the Voyager 2 spacecraft during its Neptune flyby. Even if the ngVLA could only be used for short-durations events, like 
a probe entry or particular flybys during a mission's cruise phase, we encourage NASA and the Decadal Survey to seriously consider some participation in the ngVLA.

\section{Conclusions}

To determine the composition and unravel the dynamics in the Ice Giants' atmospheres we need to map the 3D distribution of gases throughout their tropospheres (e.g., $\mathrm{CH}_{4}, \mathrm{NH}_{3}, \mathrm{H}_{2} \mathrm{~S}$ ). This requires mapping at continuum wavelengths across the $\mathrm{mm}-\mathrm{cm}$ wavelength range at high spatial resolution. In addition, high spatial and spectral resolution maps are needed of disequilibrium species in the upper troposphere/lower stratosphere to constrain the dynamics and deep water abundance (through $\mathrm{CO}$ ). We showed that the ngVLA provides the spatial resolution, spectral coverage, and sensitivity to characterize the tropospheres and (lower) stratospheres of the Ice Giants in detail. Such maps can further be obtained over decades-long timescales of all giant planets to capture changes in the atmospheres to study their long-term climate. We encourage the Decadal Survey to recognize the importance of the ngVLA to advancing NASA's goals in the Solar System.

\section{References}

1. Bolton, S.J., et al., 2017. Science 356, 821-825.

2. Cavalié, T., et al., 2020. Sp. Sci. Rev., 216, \#58.

3. de Pater, I., et al., 1991, Icarus, 91, 220-233

4. de Pater, I., et al., 2014. Icarus, 237, 211-238.

5. de Pater, I., et al., 2016. Science, 352, 1198-1201.

6. de Pater, I., et al., 2018. ASP Series: Science with a Next-Generation VLA, ed. E. J. Murphy.

7. de Pater, I., et al., 2019. Icarus, 322, 168-191.

8. Fletcher, L.N., et al., 2020a. SSRv, in Press.

9. Fletcher, L.N., et al., 2020b. JGR, in press.

10. Gulkis, S. et al., 1978. Icarus 34, 10-19.

11. Hofstadter, M., Butler, B., 2003. Icarus, 165, 168-180

12. Hsu, A.I., et al., 2019. AJ 157:152 (9pp)

13. Lazio, T.J.W., Asmar, S., 2018. ASP: Science with a Next-Generation VLA, ed. E.J. Murphy.

14. Li, C., et al., 2017. Geophys. Res. Lett. 44, 5317-5325.

15. Luszcz-Cook, S.H., de Pater, I., 2013. Icarus, 222, 379-400.

16. Marten, A. et al., 1993. Astrophys. J. 406, 285-297.

17. Molter, E.M., et al., 2020. PSJ, submitted.

18. Petigura, E.A., et al., 2013. PNAS 110, 19273-19278.

19. Roman, M.T., 2020. Astron, J., 159:45.

20. Sault, R.J., et al., 2004. Icarus, 168, 336-343.

21. Selina, R.J., et al., 2018. SPIE Astron. Telescopes \& Instrumentation, AS18, 10700-55.

22. Showman, A.P., Dowling, T.E., 2000. Science 289, 1737-1740.

23. Tollefson, J., et al., 2020. PSJ, submitted.

24. Wong, M.H., et al., 2011. Icarus 215, 211-225.

25. Young, R.E., 2003. New Astronomy Reviews 47, 1-51. 